\title{
A CONTINUOUS FUNCTION WHOSE DEVELOPMENT IN BESSEL'S FUNCTIONS IS NON-SUMMABLE OF CERTAIN ORDERS.
}

BY PROFESSOR CHARLES N. MOORE.

(Read before the American Mathematical Society, September 4, 1916.)

The first example of a continuous function whose Fourier's development diverges at one or more points was given, as is well known, by Du Bois-Reymond.* Much more recently Haar $\nmid$ showed how to construct a continuous function whose Sturm-Liouville development is divergent, and a continuous function whose development in Legendre's functions has this same property. Somewhat later Gronwall $\ddagger$ gave in explicit form a function whose development in Legendre's functions is not summable $(C k)$ for $0 \leqq k \leqq \frac{1}{2}$, at the point $x=1$.

As far as the writer is aware, no examples have thus far been given of continuous functions whose developments in Bessel's functions diverge or fail to be summable of certain orders at one or more points. In the present paper there is exhibited in explicit form a continuous function whose development in Bessel's functions of order zero is not summable $(C k)$ for $0 \leqq k<\frac{1}{2}$, at the point $x=0$. This function is analogous to a function given by Fejér\$ whose Fourier development diverges for $x=0$. The proof of the non-summability, however, follows different lines from Fejér's proof of the divergence for his example.

If we represent by $A_{r}$ the $r$ th coefficient in the development of an arbitrary function $f(x)$ in terms of Bessel's functions, we have

$$
A_{r}=\frac{\int_{0}^{1} x f(x) J_{0}\left(\lambda_{r} x\right) d x}{\int_{0}^{1} x J_{0}^{2}\left(\lambda_{r} x\right) d x},
$$

where $\lambda_{r}$ is the $r$ th root of the equation

* Abhandlungen der Bayerischen Akademie der Wissenschaften, vol. 12 (1876).

† Inaugural Dissertation, Göttingen (1909); also Math. Annalen, vol. 69 (1910).

$\ddagger$ Math. Annalen, vol. 75 (1914).

\$ Journal für die reine und angewandte Mathematik, vol. 137 (1910). 


$$
l \lambda J_{0}^{\prime}(\lambda)+h J_{0}(\lambda)=0,
$$

$l$ and $h$ being any constants not both zero. Moreover, we have for $\lambda_{r}$

$$
\lambda_{r}=(r+q) \pi+\frac{\psi(r)}{r},
$$

where $q$ is a constant and $\psi(r)$ is used to represent a function of $r$ that remains finite for all values of $r$.

Consider now the function $f(x)$ defined by the following equation

$$
f(x)=\sum_{n=1}^{\infty} \frac{\sqrt{x} \cos \left(2^{n}+q\right) \pi x}{n^{2}},
$$

where $q$ is the $q$ of (3). We will show that for the coefficients $A_{r}$ in the development of this function in terms of Bessel's functions

$$
\lim _{m \rightarrow \infty} \frac{A_{2 m}}{\left(2^{m}\right)^{k}}=\infty \quad\left(0 \leqq k<\frac{1}{2}\right) .
$$

Thus it will follow that the series

$$
A_{1}+A_{2}+A_{3}+\cdots+A_{r}+\cdots
$$

is not summable $(C k)$ for $0 \leqq k<\frac{1}{2}$, since for any series so summable the limit on the left-hand side of (5) exists and is equal to zero.* But since $J_{0}(0)=1$, the development of $f(x)$ in Bessel's functions of order zero reduces to the form (6) for $x=0$, and therefore it will have been shown that this development is not summable $(C k)$ for $0 \leqq k<\frac{1}{2}$ at the point $x=0$

It only remains then to establish the relationship (5). Since the series on the right-hand side of (4) is uniformly convergent in the interval $0 \leqq x \leqq 1$, we have from (1)

$$
A_{r}=\frac{1}{\int_{0}^{1} x J_{0}^{2}\left(\lambda_{r} x\right) d x} \sum_{n=1}^{\infty} \frac{\int_{0}^{1} x^{\frac{3}{2}} \cos \left(2^{n}+q\right) \pi x J_{0}\left(\lambda_{r} x\right) d x}{n^{2}} .
$$

From the asymptotic expansion of $J_{0}(x)$ we have

$$
J_{0}(\lambda x)=\sqrt{\frac{2}{\pi \lambda x}} \cos \left(\lambda x-\frac{\pi}{4}\right)+\frac{\psi(\lambda x)}{(\lambda x)^{\frac{3}{2}}} .
$$

* Cf. Chapman, Proc. London Math. Society, ser. 2, vol. 9 (1911), p. 379. 
Making use of (8), we see that the general term of the series on the right-hand side of (7) may be written in the form

$$
\begin{aligned}
\frac{1}{n^{2}} \sqrt{\frac{2}{\pi \lambda_{r}}} \int_{0}^{1} x \cos \left(\lambda_{r} x-\frac{\pi}{4}\right) \cos \left(2^{n}+q\right) \pi x d x \\
+\frac{1}{n^{2} \lambda_{r}^{\frac{3}{2}}} \int_{0}^{1} \cos \left(2^{n}+q\right) \pi x \psi\left(\lambda_{r} x\right) d x .
\end{aligned}
$$

If now we set $r=2^{m}$ and make use of the relation (3), it is readily seen that the sum of the series whose general term is the second term of (9) is of the form

$$
O\left(\frac{1}{\lambda_{r^{\frac{3}{2}}}}\right)=O\left(\frac{1}{\sqrt{\lambda_{r}}} \cdot \frac{1}{2^{m}}\right)=o\left(\frac{1}{\sqrt{\lambda_{r}}} \cdot \frac{1}{m^{2}}\right),
$$

where we have used $O(x)$ to represent any function which after division by $x$ remains finite as $x$ becomes infinite, and $o(x)$ to represent any function which after division by $x$ approaches zero as $x$ becomes infinite.*

We have further to consider the series whose general term is the first term of (9). Making use of (3) we may write

$$
\begin{aligned}
& \cos \left(\lambda_{r} x-\frac{\pi}{4}\right) \\
& =\cos \frac{\pi}{4} \cos (r+q) \pi x+\sin \frac{\pi}{4} \sin (r+q) \pi x \\
& +\cos \left\{(r+q) \pi x-\frac{\pi}{4}+\frac{\psi(r)}{r} x\right\}-\cos \left\{(r+q) \pi x-\frac{\pi}{4}\right\},
\end{aligned}
$$

and the last two terms on the right-hand side of (11) may be written in the form

$$
-\frac{\psi(r)}{r} x \sin \left\{(r+q) \pi x-\frac{\pi}{4}+\theta\right\} \quad\left(|\theta|<\left|\frac{\psi(r)}{r} x\right|\right) .
$$

Substituting from (11) in the first term of (9) and making use of (12), we reduce this term to the form

$$
\frac{1}{n^{2} \sqrt{\pi \lambda_{r}}} \int_{0}^{1} x \cos \left(2^{m}+q\right) \pi x \cos \left(2^{n}+q\right) \pi x d x
$$

* The use of $O$ in this sense is due to Bachmann. Cf. Analytische Zahlentheorie, vol. 2, p. 401. The corresponding use of $o$ is due to Landau. Cf. Handbuch der Lehre von der Verteilung der Primzahlen, vol. 1, p. 61. 


$$
\begin{array}{r}
+\frac{1}{n^{2} \sqrt{\pi \lambda_{r}}} \int_{0}^{1} x \sin \left(2^{m}+q\right) \pi x \cos \left(2^{n}+q\right) \pi x d x \\
-\frac{\psi\left(2^{m}\right)}{2^{m} \cdot n^{2}} \cdot \sqrt{\frac{2}{\pi \lambda_{r}}} \int_{0}^{1} x^{2} \sin \left\{\left(2^{m}+q\right) \pi x\right. \\
\left.-\frac{\pi}{4}+\theta\right\} \cos \left(2^{n} x+q\right) \pi x d x .
\end{array}
$$

The last term in (13) is obviously the general term of a series whose sum is of the form (10). Consider next the first term in (13). If $n \neq m$, the integral in this term reduces to the form

$$
\begin{aligned}
& \frac{\cos \left(2^{m}+2^{n}+2 q\right) \pi-1}{2\left(2^{m}+2^{n}+2 q\right)^{2} \pi^{2}}+\frac{\sin \left(2^{m}+2^{n}+2 q\right) \pi}{2\left(2^{m}+2^{n}+2 q\right) \pi} \\
& \quad+\frac{\cos \left(2^{m}-2^{n}\right) \pi-1}{2\left(2^{m}-2^{n}\right)^{2} \pi^{2}}+\frac{\sin \left(2^{m}-2^{n}\right) \pi}{2\left(2^{m}-2^{n}\right) \pi}
\end{aligned}
$$

This quantity is obviously less in absolute value than $K$ / $\left|2^{m}-2^{n}\right|$, where $K$ is a positive constant. Moreover, for all values of $n<m$

$$
2^{m}-2^{n}>2^{n}\left(2^{m-n}-1\right) \geqq 2^{n} \cdot 2^{m-n-1}=2^{m-1},
$$

and for all values of $n>m$

$$
2^{n}-2^{m} \geqq 2 \cdot 2^{m}-2^{m}=2^{m} \text {. }
$$

Hence it follows that $K /\left|2^{m}-2^{n}\right|<K_{i} / 2^{m-1}$, and therefore the sum of all the terms of the form of the first term in (13) for which $n \neq m$ is of the form $O\left(1 /\left(\sqrt{\lambda_{r}} \cdot 2^{m-1}\right)\right)$ and consequently of the form of the last member of (10).

If $n=m$, the integral in the first term of (13) reduces to

$$
\frac{\cos 2\left(2^{m}+q\right) \pi-1}{8\left(2^{m}+q\right)^{2} \pi^{2}}+\frac{\sin 2\left(2^{m}+q\right) \pi}{4\left(2^{m}+q\right) \pi}+\frac{1}{4},
$$

and hence for this value of $n$ the first term of (13) takes the form

$$
o\left(\frac{1}{\sqrt{\lambda_{r}}} \cdot \frac{1}{m^{2}}\right)+\frac{1}{4 \sqrt{\pi} \sqrt{\lambda_{r} m^{2}}} .
$$

Combining this with the previous result, we see that the series 
of which the first term of (13) is the general term has a sum which is of the form (14).

We have finally to discuss the second term of (13). The integral in this term may for $n \neq m$ be reduced to the form

$$
\begin{aligned}
& \frac{\sin \left(2^{m}+2^{n}+2 q\right) \pi}{2\left(2^{m}+2^{n}+2 q\right)^{2} \pi^{2}}-\frac{\cos \left(2^{m}+2^{n}+2 q\right) \pi}{2\left(2^{m}+2^{n}+2 q\right) \pi} \\
& +\frac{\sin \left(2^{m}-2^{n}\right) \pi}{2\left(2^{m}-2^{n}\right)^{2} \pi^{2}}-\frac{\cos \left(2^{m}-2^{n}\right) \pi}{2\left(2^{m}-2^{n}\right)} .
\end{aligned}
$$

For $n=m$ it takes the form

$$
\frac{\sin 2\left(2^{m}+q\right) \pi}{8\left(2^{m}+q\right)^{2} \pi^{2}}-\frac{\cos 2\left(2^{m}+q\right) \pi}{4\left(2^{m}+q\right) \pi} .
$$

Hence the series whose general term is the second term of (13) has a sum which is of the form of the last member of (10).

We have now seen that the series whose general terms are the second and third terms of (13), respectively, have sums that are of the form of the last member of (10), whereas the series whose general term is the first term of (13) has a sum that is of the form (14). Hence the series whose general term is (13), or the first term of (9), has a sum which is of the form (14). Since, moreover, the series whose general term is the second term of (9) has a sum which is of the form (10), it follows that the series whose general term is (9), that is, the series in the right-hand member of (7), has a sum which is of the form (14). As the other factor of the right-hand member of (7) is of the form $C \lambda_{r}+o\left(\lambda_{r}\right)$, where $C$ is a positive constant, it follows that

$$
\begin{aligned}
A_{r}=C_{1} \frac{\sqrt{\lambda_{r}}}{m^{2}}+o\left(\frac{\sqrt{\lambda_{r}}}{m^{2}}\right)=C_{2} \frac{\sqrt{r}}{m^{2}} & +o\left(\frac{\sqrt{r}}{m^{2}}\right) \\
& =C_{2} \frac{\sqrt{2^{m}}}{m^{2}}+o\left(\frac{\sqrt{2^{m}}}{m^{2}}\right),
\end{aligned}
$$

where $C_{1}$ and $C_{2}$ are positive constants and we make use of the fact that we have chosen $r=2^{m}$.

It follows from (15) that $A_{r}$ satisfies the relationship (5), and hence as pointed out before, the development in Bessel's functions of order zero of the function $f(x)$ defined by (4) is not summable $(C k)$ for $0 \leqq k<\frac{1}{2}$ at the point $x=0$.

UnIVERSiTY OF CinCINNATI. 